\title{
COMPUTATIONALLY EFFICIENT MODELING OF DELAMINATION BEHAVIOR IN LAMINATED COMPOSITES
}

\author{
Khairul Anam $^{1,2, *}$, Heinz E. Pettermann ${ }^{1}$ and Melanie Todt ${ }^{1}$ \\ ${ }^{1}$ Institute of Lightweight Design and Structural Biomechanics, TU Wien, Vienna, Austria \\ ${ }^{2}$ On leave from Department of Mechanical Engineering, Brawijaya University, Malang, Indonesia \\ *anam@ilsb.tuwien.ac.at, pettermann@ilsb.tuwien.ac.at, mt@ilsb.tuwien.ac.at
}

The simulation of progressive delamination in multilayered laminate components requires considerable computational resources. A numerical efficient approach has been employed earlier [1], utilizing shell elements to model the individual plies and cohesive zone elements (CZE) in-between. In the present paper, this modeling strategy is adopted and various choices of element types to represent the plies are studied. Shell elements and continuum shell elements are combined with CZEs in different ways, Figure 1a-c, and conventional modeling based on continuum solid elements, Figure 1d, is used as reference solution. The delamination behavior of these models is quantitatively compared in terms of nonlinear load-displacement curves, delamination area, and energy dissipation. Computation time is also assessed for these approaches as being considered for simulations of large scale laminated composite components.

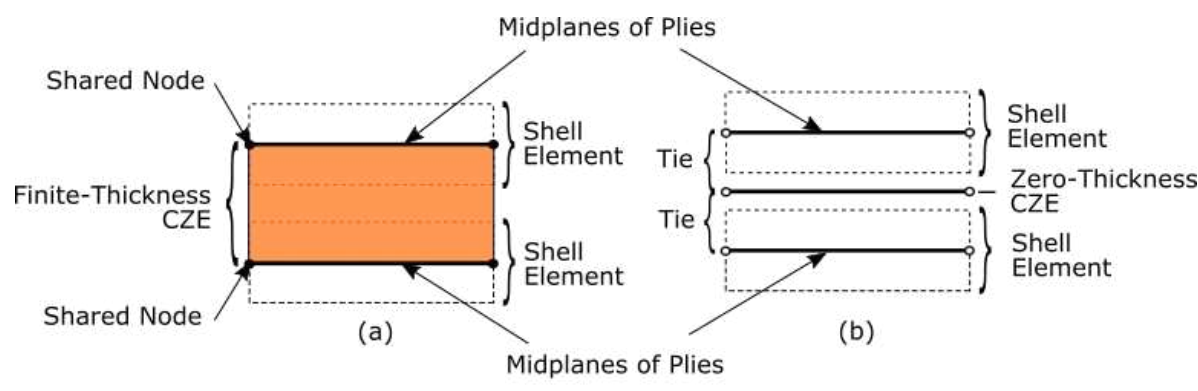

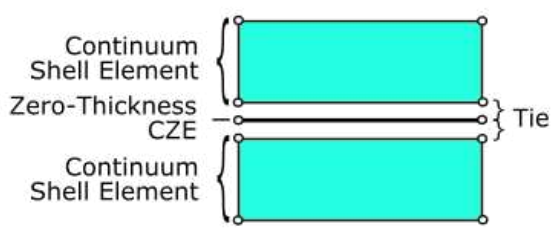

(c)

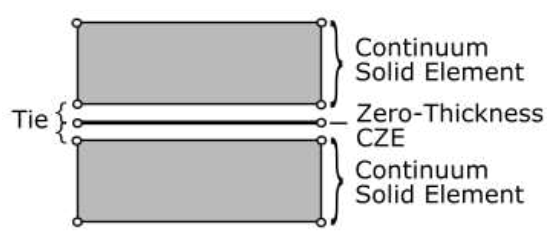

(d)

Figure 1: Sketch of the modeling strategies of one interface and the adjacent plies; (a) shell elements with finitethickness CZE, (b-d) shell, continuum shell, and continuum solid elements with zero-thickness CZE, respectively.

\section{Acknowledgements}

The funding of KA by the Indonesian Ministry of Education and Culture (KEMDIKBUD) and Österreichs Agentur für Bildung und Internationalisierung $(\mathrm{OeAD}-\mathrm{GmbH})$ in cooperation with ASEAN European Academic University Network (ASEA-UNINET) under Indonesia-Austria Scholarship Programme is gratefully acknowledged.

\section{References}

[1] M. Schwab, H. Pettermann (2016) Modeling and simulation of damage and failure in large composite components subjected to impact loads. Composite Structures, 158, 208-216. 